Cahiers $d u$ MONDE RUSSE

\section{Cahiers du monde russe}

Russie - Empire russe - Union soviétique et États indépendants

$44 / 1 \mid 2003$

Varia

\title{
L'image de Moscou entre la description standardisée des Lumières et la recherche de la singularité russe
}

La topographie médicale (1803) de Engelbrecht Wichelhausen

\section{Martin DINGES}

\section{OpenEdition}

\section{Journals}

Édition électronique

URL : https://journals.openedition.org/monderusse/8598

DOI : $10.4000 /$ monderusse.8598

ISSN : $1777-5388$

\section{Éditeur}

Éditions de l'EHESS

Édition imprimée

Date de publication : 1 janvier 2003

Pagination : $35-56$

ISBN : 2-7132-1812-8

ISSN : $1252-6576$

\section{Référence électronique}

Martin DINGES, «L'image de Moscou entre la description standardisée des Lumières et la

recherche de la singularité russe ", Cahiers du monde russe [En ligne], 44/1 | 2003, mis en ligne le 01 janvier 2007, consulté le 04 septembre 2022. URL : http://journals.openedition.org/monderusse/8598 ; DOI : https://doi.org/10.4000/monderusse.8598 
chercher : repérer : avancer

Cet article est disponible en ligne à l'adresse :

http://www.cairn.info/article.php?ID REVUE=CMR\&ID NUMPUBLIE=CMR 441\&ID ARTICLE=CMR 4410035

\title{
L'image de Moscou entre la description standardisée des Lumières et la recherche de la singularité russe. La topographie médicale (1803) de par Martin DINGES
}

\section{Editions de I'EHESS | Cahiers du monde russe}

\author{
2003/1 - Vol 44 \\ ISSN 1252-6576 | ISBN 2713218128 | pages 35 à 56
}

Pour citer cet article :

-DINGES M. , L'image de Moscou entre la description standardisée des Lumières et la recherche de la singularité russe. La topographie médicale (1803) de Engelbrecht Wichelhausen, Cahiers du monde russe 2003/1, Vol 44, p. 3556 .

Distribution électronique Cairn pour les Editions de l'EHESS.

(C) Editions de l'EHESS. Tous droits réservés pour tous pays.

La reproduction ou représentation de cet article, notamment par photocopie, n'est autorisée que dans les limites des conditions générales d'utilisation du site ou, le cas échéant, des conditions générales de la licence souscrite par votre établissement. Toute autre reproduction ou représentation, en tout ou partie, sous quelque forme et de quelque manière que ce soit, est interdite sauf accord préalable et écrit de l'éditeur, en dehors des cas prévus par la législation en vigueur en France. Il est précisé que son stockage dans une base de données est également interdit. 


\title{
L'IMAGE DE MOSCOU ENTRE LA DESCRIPTION STANDARDISÉE DES LUMIĖRES ET LA RECHERCHE DE LA SINGULARITÉ RUSSE
}

\author{
La topographie médicale (1803) \\ de Engelbrecht Wichelhausen
}

\section{Introduction : recherches sur l'image de la Russie en Allemagne}

L'image de l'autre est un champ de recherche bien établi qui intéresse de multiples disciplines scientifiques. L'image que certains peuples se font des autres peuples est un des sujets de recherche particulièrement bien étudié depuis longtemps. L'histoire du XXe siècle - surtout dans sa première moitié - , qui a fait la collecte des préjugés aussi bien nationalistes qu'impérialistes et racistes sous toutes leurs formes, a particulièrement contribué à la reconstitution des stéréotypes que chaque peuple projetait sur sa vision des autres. Dans ce contexte s'est aussi posée la question des fondements historiques de ces stéréotypes et de l'importance des formes de diffusion auxquelles ils ont donné lieu, pouvant aller parfois jusqu'à la propagande intentionnelle des États. Les recherches de la deuxième moitié du siècle, qui sont inspirées en Europe occidentale par une tentative, plutôt réussie, de rapprochement entre les peuples, ont montré que les distorsions des perceptions mutuelles étaient loin d'être insignifiantes. Le projet de les faire disparaître s'est révélé trop optimiste. Il paraît beaucoup plus fructueux de comprendre les "préjugés », leur devenir et leur manière de fonctionner pour en tenir pleinement compte en acceptant les distances existant entre les peuples ${ }^{1}$.

1. Je remercie Michael Stolberg, Mauricio Gribaudi et les participants au séminaire du Centre d'études du Monde russe, soviétique et post-soviétique de l'EHESS pour leurs commentaires et suggestions. Jürgen Osterhammel, «Distanzerfahrung. Darstellungsweisen des Fremden im 18. Jahrhundert », in Hans-Joachim König, Wolfgang Reinhard, Reinhard Wendt, eds, Der europäische Beobachter außereuropäischer Kulturen, Berlin, 1989, p. 9-42, 20. 
La brutale expérience de la Deuxième Guerre mondiale a donné naissance à une vaste littérature scientifique portant sur l'image que les Allemands se font des Russes. Les premiers cherchaient à comprendre les antécédents historiques de l'antibolchevisme ${ }^{2}$, les seconds reconstruisaient l'image des Russes développée en Allemagne depuis le Moyen $\hat{A}_{\text {ge }}{ }^{3}$ et ces derniers voulaient faire connaître et accepter la tradition de la « fraternité germano-slave » dans le cadre des réorientations politiques de la République démocratique allemande ${ }^{4}$. Depuis les années 1980, Lev Kopelev a coordonné un projet russo-allemand à Wuppertal visant à explorer systématiquement les images que les Russes avaient des Allemands et inversement ${ }^{5}$. Cette double perspective permettait ainsi de poser les jalons intellectuels d'une réconciliation fondée sur la reconnaissance de l'importance de la vision de chacun, et il est apparu à l'évidence que l'image de l'autre est souvent beaucoup plus révélatrice des désirs et des problèmes de celui qui la produit qu'elle ne livre d'informations sur l'autre' ${ }^{6}$.

Nous savons bien, maintenant, que les images « des Russes » sont (et étaient) très différentes, selon la source considérée, allemande, française ou anglaise - même si beaucoup de textes ont été traduits ${ }^{7}$. Des images différentes pouvaient être en concurrence au même moment ${ }^{8}$. Les images changeaient parfois de décennie en décennie et souvent en étroite relation avec l'histoire politique - par exemple autour de 1763, $1812,1917,1941$, pour ne donner que quelques années clés ${ }^{9}$. On a aussi reconnu l'importance du rôle des médias véhiculant ces images : en se limitant aux exemples empruntés au XVIII siècle, les récits de voyageurs (volontaires, ou involontaires comme les prisonniers de guerre $)^{10}$, les rapports des diplomates, les volumineuses

2. Rudolf Jaworski, «Osteuropa als Gegenstand historischer Stereotypenforschung », Geschichte und Gesellschaft, 13, 1987, p. 63-76.

3. Dieter Groh, Rußland und das Selbstverständnis Europas. Ein Beitrag zur europäischen Geistesgeschichte, Neuwied, 1961.

4. Michael Schippan, « Deutsche in Moskau und Sankt Petersburg in der Historiographie der DDR », Nordost-Archiv, N.F. 3, 1994, p. 193-200 ; pour le concept de réciprocité germanoslave d'Eduard Winters, voir p. 200.

5. Lew Kopelew, ed., West-östliche Spiegelungen, deux séries, plusieurs volumes, Munich, 1985, sq.

6. Thorsten Sadowsky (in Reisen durch den Mikrokosmos. Berlin und Wien in der bürgerlichen Reiseliteratur um 1800, Hambourg, 1998, p. 43, 46) place cette idée au centre de sa recherche ; J. Osterhammel, in H. J. König, W. Reinhard, R. Wendt, eds, Der europäische Beobachter..., op. cit., passim.

7. Inge Hanslik, Das Bild Rußlands und Polens im Frankreich des 18. Jahrhunderts, Francfort/ M.- Berne, 1985 ; cf. par exemple sur les traductions des récits de voyage, Gert Robel, «Berichte über Rußlandreisen », in Mechthild Keller, ed., Russen und Rußland aus deutscher Sicht. 18. Jahrhundert : Aufklärung, Munich, 1987, p. 228, 234 et passim.

8. Jürgen Kämmerer, « Theorie und Empirie. Rußland im Urteil aufgeklärter Philosophen und Reiseschriftsteller », in B. I. Krasnobaev, Gert Robel, Herbert Zeman, eds, Reisen und Reisebeschreibungen im 18. und 19. Jahrhundert als Quellen der Kulturbeziehungsforschung, Berlin, 1980,p. 331-351.

9. Cf. la répartition des chapitres du livre de Groh ; Erhard Hexelschneider, Kulturelle Begegnungen zwischen Sachsen und Rußland 1790-1849, Cologne-Weimar, 2000, passim et p. 91 sq.

10. G. Robel, « Berichte über Rußlandreisen », art. cit., p. 216-247. 
descriptions des explorateurs scientifiques et des spécialistes de «statistique politique », les textes littéraires et philosophiques, les journaux livrent, tous, des images assez diverses - y compris à l'intérieur d'un même genre littéraire ${ }^{11}$.

Ces genres littéraires ont été en partie inventoriés et rassemblés en un corpus de textes qui permet de mieux saisir leurs filiations. On a décrit et classé leurs contenus : les critères des chercheurs étaient par exemple la russo- (ou slavo-)philie ou la russo- (ou slavo-)phobie. On a aussi étudié la relation entre différents jugements exprimés sur la Russie et différents stades du mouvement des Lumières. Ces classifications extérieures sont utiles. Le classement des textes qu'elles assurent, conformément à leur fonction, tend à simplifier chaque texte en gommant les tensions et contradictions internes qu'il recèle inévitablement. Celles-ci me paraissent pourtant tellement plus intéressantes que ce sont elles qui seront traitées dans cet article qui tentera de répondre à la question « comment fonctionne un texte des Lumières ? ». Je m'intéresserai donc à sa composition, à ses topiques, à sa technique narrative, à ses présupposés et à ses formes d'argumentation, de préférence au contenu du texte en soi qui est trop souvent pris pour argent comptant. Pour donner un exemple, ce n'est pas tant la question de savoir comment les Russes mangeaient et si leur façon de manger était considérée comme saine ou malsaine par l'auteur qui m'intéresse, mais dans quel contexte cet argument était utilisé et à quelle fin.

Le mouvement des Lumières se concevant comme un acteur de la lutte contre les préjugés, engagé dans un combat pour améliorer le monde, il est passionnant de reconstruire ses grilles de lecture de façon à pouvoir apprécier si elles ont la capacité d'éviter les préjugés ou, au contraire, si elles ne font que contribuer à en produire d'autres. Autrement dit, comment le texte considéré donne-t-il à voir l'autre personne, groupe social ou peuple, en l'occurrence les Russes ? Quelles stratégies utilise-t-il pour fonder ses énoncés ? Est-il possible d'appliquer des principes « universels » tout en décrivant les particularités russes sans tomber dans des contradictions? Si ces contradictions existent, l'auteur s'en rend-il compte et cherche-t-il à les résoudre?

\section{La source, son auteur et son contexte}

Pour cette recherche, il est préférable de choisir un texte riche, de « haut niveau », « scientifique » et solide pour saisir les Lumières à travers un de ses «produits» «le plus à la pointe ». J'ai choisi la «topographie médicale» de Moscou du médecin allemand Engelbrecht Wichelhausen (1760-1814) publiée en 1803. Le

11. Voir, par exemple, E. Hexelschneider, Kulturelle Begegnungen..., op. cit., p. 92-110, et les différents articles dans M. Keller, ed., Russen und Rußland.., op. cit., par exemple Harm Klueting, « Rußland in den Werken deutscher Statistiker des 18. Jahrhunderts », p. 248-260 sur les statisticiens ; pour le théâtre comme « média de masse », voir Svetlana Vladimirova, « Das Rußlandbild im österreichischen Drama am Ende des 18. Jahrhunderts », in Johann Holzner, Stefan Simonek, eds, Rußland - Österreich : literarische und kulturelle Wechselwirkungen, Berne, 2000,p. 27-36, 27. 
titre complet de l'ouvrage donne une première idée de son contenu : Esquisse d'une peinture de Moscou du point de vue du climat, de la culture, des mœurs, du genre de vie, des coutumes mais surtout des conditions statistiques, physiques et médicales $^{12}$. Le texte est parfaitement représentatif de l'effort des Lumières dans le domaine de la médecine, visant à accroître la connaissance des questions médicales parmi les décideurs et à sensibiliser les populations ${ }^{13}$. Le livre de Wichelhausen avec ses 371 pages (in octavo) de texte s'adresse certainement au public cultivé. Le titre indique que cette topographie, comme les autres, a une vision très large du «médical ». Elle englobe autant l'éventail des anciennes descriptions des peuples (étrangers) que la plus récente méthode statistique qui est mise en avant dès le titre. Dans ce genre, l'intérêt se porte sur l'habitat, sur les modes de vie comme sur les manières d'habiter aussi bien que sur l'environnement naturel et la constitution politique, parce qu'on considère que celle-ci a une grande influence sur l'état moral du peuple. La partie statistique concerne la démographie. Les questions médicales peuvent concerner autant l'offre des professions médicales que la qualité chimique de l'eau. Une des visées importantes de ces textes est de présenter les médecins comme des spécialistes de l'environnement, de la santé et de l'hygiène pour leur donner une importance professionnelle accrue auprès des pouvoirs publics ${ }^{14}$. Ces médecins, auteurs de topographies, faisaient souvent carrière après la publication de leurs textes ${ }^{15}$. On peut supposer que Wichelhausen dédicaça sa topographie au margrave de Bade dans ce même espoir d'avancement professionnel.

12. Engelbert Wichelhausen, Züge zu einem Gemählde von Moskwa in Ansicht auf Klima, Cultur, Sitten, Lebensart, Gebräuche, vorzüglich aber statistische, physische und medicinische Verhältnisse, Berlin, 1803.

13. Sur le genre «topographie médicale » cf. Jan Brügelmann, Der Blick des Arztes auf die Krankheit im Alltag 1779-1850, diss. phil. Freie Universität Berlin 1982, spéc. p. 16, 19 (définition), 26 sq., 47 sq. Un exemple bien étudié : Barbara Becker-Jákli, ed., Köln um 1825 - ein Arzt sieht seine Stadt. Die medizinische Topographie der Stadt Köln von Dr. Bernard Elkendorf, Cologne, 1999 ; sur la France voir la plus récente étude comportant une vue comparative sur les ingénieurs: Sabine Barles, La ville délétère. Médecins et ingénieurs dans l'espace urbain, $X V I I I^{e}-X I X^{\mathrm{e}}$ siècle, Paris, 1999, chap. 1 ; pour un cas individuel, voir Jean-Pierre Goubert, Médecins d'hier - médecins d'aujourd'hui. Le cas du docteur Lavergne (1756-1831), Paris, 1992. Concernant un contexte plus large, cf. Conevery Bolton Valenčius, « Histories of medical geography », in Nicolaas A. Rupke, ed., Medical geography in historical perspective, Londres, 2000, p. 3-28, 7-15; sur « Medizinische Aufklärung » - le mouvement des Lumières en médecine, cf. Francisca Loetz, «'... nicht durch Einschreiten oder Zwang, sondern durch Belehrung und Warnung': Polyvalenzen als Modellelemente zur Erforschung der Volksaufklärung », in Anne Conrad, Arno Herzig, Franklin Kopitzsch, eds, Das Volk im Visier der Aufklärung. Studien zur Popularisierung der Aufklärung im späten 18. Jahrhundert, Hambourg, 1998, p. 239-259 et Martin Dinges, «Medizinische Aufklärung bei Johann Georg Zimmermann. Zum Verhältnis von Macht und Wissen bei einem Arzt der Aufklärung », in Martin Fontius, Helmut Holzhey, eds, Schweizer im Berlin des 18. Jahrhunderts, Berlin, 1996, p. 137-150.

14. Sur le contexte propre à la médecine, cf. Martin Dinges, «'Medicinische Policey' zwischen Heilkundigen und 'Patienten' (1750-1830) », in Karl Härter, ed., Policey und frühneuzeitliche Gesellschaft, Francfort/M., 2000, p. 263-295, avec une bibliographie sur cette étape de la professionnalisation des médecins. Cf. pour la France, Patrice Bourdelais, ed., Les hygiénistes : enjeux, modèles, pratiques (XVIII ${ }^{\mathrm{e}}-\mathrm{X} \mathrm{X}^{\mathrm{e}}$ siècles), Paris, 2001.

15. J. Brügelmann, Der Blick des Arztes..., op. cit., p. 41. 
Né, le 27 octobre 1760, dans une famille de commerçants de Brême, il termina ses études à la fameuse université de Göttingen/Goettingue où il soutint sa thèse en $1783^{16}$. À cette époque Göttingen était la faculté de médecine allemande qui encourageait le plus le renouveau de l'observation empirique en médecine ; c'est elle qui donna l'impulsion aux topographies médicales ${ }^{17}$. Wichelhausen devenait la même année (1783) médecin en titre de la ville de Brême et professeur de médecine au lycée municipal. Ensuite il répondit à l'appel de Catherine II aux médecins allemands en 1786, devenant médecin officiel de la province de Saint-Pétersbourg, et en 1788 médecin du cabinet et assesseur au Collège [administration supérieure] ${ }^{18}$. En 1790 il avait obtenu la permission de quitter Pétersbourg pour Moscou, où il exerça sa profession de médecin jusqu'en 1793 pour voyager par la suite en Suisse, en Italie et en France. Après quelques années à Hambourg (1796-1797) et dans d'autres villes allemandes, il se fixa à Mannheim en 1805 où il mourut en 1814.

Pendant ses années à Moscou, Wichelhausen tint un journal, qu'il utilisa pour la rédaction de sa topographie. Il en tire par exemple ses informations sur les épidémies des années 1790 et $1791^{19}$. Avant de publier sa topographie médicale de Moscou, Wichelhausen a beaucoup voyagé à travers des pays qui préparaient des réformes du système de santé publique et de formation des médecins ${ }^{20}$. En même temps, ces voyages ont dû aiguiser son regard comparatif. Wichelhausen n'est donc pas seulement un témoin occulaire, mais aussi un auteur auquel on peut supposer une compétence multiculturelle.

Le texte, au-delà de ses caractéristiques externes déjà évoquées (gros livre de 371 pages), comporte de multiples éléments d'auto-légitimation, surtout scientifiques. Sur la page de titre Wichelhausen énumère toutes ses fonctions antérieures au service de sa Majesté de Russie, du titre de professeur de médecine jusqu'à celui de

16. August Hirsch, Biographisches Lexikon der hervorragenden Ärzte aller Zeiten und Völker, vol. 5, 3e édition, Munich, (1962 [1934]), p. 923. La dissertation portait sur la «Phthisi pituitosa »; on a procédé à des recherches dans différentes archives allemandes et constaté que le maximum d'information biographique se trouve dans Heinz E. Müller-Dietz, Ärzte zwischen Deutschland und Rußland, Stuttgart-Iéna, 1995, p. 48. Deux autographes de lettres (provenant de la bibliothèque d'État de Berlin) se trouvent dans la bibliothèque de Cracovie.

17. Entre 1783 et 1788 , six futurs auteurs de topographies médicales y passaient leur doctorat, voir J. Brügelmann, Der Blick des Arztes..., op. cit., p. 38.

18. Sur ces « recrues allemandes », voir Heinz E. Müller-Dietz, « Die medizinischen 'Rekruten' Katharinas II. », in Helmut Reinalter, ed., Gesellschaft und Kultur Mittel-, Ost- und Südosteuropas im 18. und beginnenden 19. Jahrhundert, Francfort/M., 1994, p. 167-177 et Martin Dinges, « Nemeckie vrači v Rossii vtoroj poloviny XVIII veka : konflikt kul'tur », in : S. J. Karp, ed., Russkie i Nemcy v XVIII veke. Vstreča kul'tur, Moscou, 2000, p. 160-189 (voir aussi en allemand : «Kann man medizinische Aufklärung importieren ? Kulturelle Probleme im Umfeld deutscher Ärzte in Rußland in der zweiten Hälfte des 18. Jahrhunderts », in Mathias Beer, Dittmar Dahlmann, eds, Migration nach Ost- und Südosteuropa vom 18. bis zum Beginn des 19. Jahrhunderts. Ursachen, Formen, Verlauf, Ergebnis, Stuttgart, 1999, p. 209-234.

19. E. Wichelhausen, Züge zu einem Gemählde von Moskwa..., op. cit., p. XII ; la localisation des citations du livre de Wichelhausen sera par la suite indiquée directement dans le texte, uniquement par le numéro de la page.

20. Il en tira d'autres publications sur des moulages de Florence (1798) et des « lettres d'un médecin » (1796). Il commença ses publications en Russie par un texte sur la maladie de Paul de Gagarin (1790). 
médecin de cabinet en passant par celui d'assesseur du Collège impérial russe. Le lecteur doit être impressionné par tant de compétence, d'expérience et par la position hiérarchique de l'auteur.

Wichelhausen décrit sa propre intention : il publie ce texte pour «contrer les préjugés enracinés des étrangers sur la Russie et pour aider à l'abolition de maints abus, qui empêchent un bon état de santé général et le bien-être des habitants de Moscou » (p. IX). Ces préjugés seraient aussi un obstacle à l'immigration (p. X), qu'il veut promouvoir grâce à sa publication ${ }^{21}$. Même les descriptions très récentes de Moscou seraient pleines d'erreurs. Leurs auteurs - particulièrement les (voyageurs) Français - utiliseraient « leurs propres mœurs comme mesure et échelle de leur description de la Russie ${ }^{22}$. Ce serait une manière de réfléchir hautement « antiphilosophique » (p. IX) et leurs textes ne vaudraient pas grand chose. Wichelhausen, en revanche, ayant vécu sept ans en Russie dont quatre à Moscou, saurait de quoi il parle. Dénonçant le manque total d'une vraie topographie médicale, Wichelhausen souligne qu'il s'engage contre le triste sort des serfs et se félicite « d'avoir défendu en homme juste la cause des opprimés » (XIII). Sa critique des défauts de la santé publique à Moscou serait une preuve de son patriotisme (XII).

Cette introduction contient toutes les bonnes intentions et la rhétorique habituelle des Lumières: Wichelhausen souligne que son savoir se base sur sa propre expérience, qui - longue de plusieurs années - n'est pas superficielle et a été méthodiquement enrichie et consignée par écrit dans ses journaux - référence aux carnets de voyage des expéditions scientifiques. Wichelhausen présente son œuvre comme fondatrice d'un genre littéraire établi sur la base de «véritables connaissances $»^{23}$. Il le présente comme philosophique - donc sans préjugé et ayant vocation pour les idées universalistes ${ }^{24}$. Il valorise sa critique comme patriotique et se pose en avocat en lutte pour l'émancipation de la majorité asservie du peuple russe. Il se veut aussi un partisan des améliorations pratiques.

Il est intéressant que Wichelhausen ajoute à son discours une pointe anti-française destinée à faire paraître notre auteur allemand comme un meilleur représen-

21. Les traductions des citations sont de M. D. Une autre source de renseignement sur les expériences des spécialistes en Russie est constituée par les lettres-circulaires qui étaient utilisées couramment; voir par exemple Heinz E. Müller-Dietz, «Deutsche Gelehrte erleben Rußland », in Mechthild Keller, ed., Russen und Rußland aus deutscher Sicht. 19. Jahrhundert : Von der Jahrhundertwende bis zur Reichsgründung (1800-1871), Munich, 1992, p. 151-162, 161.

22. D'autres remarques polémiques contre les concurrents français suivent, cf. p. XII. La critique des textes des voyageurs est topique, cf. par exemple Hubertus Neuschäffer, «Unterschlagene Machtpolitik - Aufklärer und Aufklärung im Baltikum zur Zeit Katharinas II. », in M. Keller, ed ., Russen und Rußland aus deutscher Sicht. 18. Jahrhundert..., op. cit., p. 396-426, sur Hupel, voir p. 410, 412.

23. Sur cette rhétorique courante, voir H. Neuschäffer, « Unterschlagene Machtpolitik... », art. cit.

24. Pour établir la comparaison avec Storch, voir Annelies Grasshoff, « Heinrich Storch (17661835). Wissenschaftliche Statistik und russische Literaturgeschichtsschreibung am Ende des 18. und Beginn des 19. Jahrhunderts », in H. Reinalter, ed., Gesellschaft und Kultur..., op. cit., p. 109-116, 114 . 
tant du mouvement international des Lumières. Comme il a eu une expérience personnelle de la concurrence avec des médecins français et avec leurs écrits, ses ressentiments (!) sont fondés autant sur le plan professionnel que national, tout comme ils sont la manifestation d'une rhétorique de la prudence face à des lecteurs critiques de la Révolution française et face à la censure allemande et russe ${ }^{25}$. Sa remarque s'insère par ailleurs bien dans la conjoncture politique du moment - la Russie au début (1801) du règne d'Alexandre Ir ${ }^{26}$. Elle reflète enfin l'image que les Lumières allemandes avaient d'elles-mêmes, à savoir qu'elles étaient les meilleures représentantes du mouvement qu'elles croyaient devoir parachever en empruntant une voie plus raisonnable que la révolution ${ }^{27}$.

À l'intérieur du texte principal, il existe des indices supplémentaires qui permettent d'identifier le statut de ce texte. Wichelhausen se caractérise par exemple comme un «médecin rationnel » (94). Il fait montre d'une indéniable connaissance des plantes dont il nous livre de longues listes qui démontrent ainsi sa compétence botanique (148-204). Il fait appel à ses connaissances en chimie pour décrire les eaux de Moscou (78), il décrit une méthode d'évaporation pour les analyses de l'eau qu'il a lui-même mise au point (70) et il lutte contre les idées fausses sur le climat de Moscou en utilisant des données de températures mesurées en degrés Réaumur et présentées sous la forme de tableaux scientifiques (101). Ces tableaux - comme les tableaux démographiques dans un autre chapitre (229) - correspondent à une certaine rhétorique du texte scientifique. Wichelhausen les introduit dans sa bataille «contre les préjugés de voyageurs non informés » (112), dont il se distingue ainsi autant par les termes utilisés que par les modes de représentation ${ }^{28}$. Le statut quasi scientifique du texte est aussi confirmé par l'usage des notes en bas de pages. Wichelhausen les ajoute à chaque fois qu'il conteste l'opinion d'un auteur, pratique qui n'était pas encore très courante à l'époque. On peut donc considérer la présence de ces notes comme une autre forme de stratégie de légitimation scientifique.

Ces notes permettent par ailleurs de connaître au moins les sources avouées de Wichelhausen, qui sont toutes des publications récentes et de haut niveau : il s'agit d'abord de la compilation des auteurs du XVI ${ }^{\mathrm{e}}$ au XVIII ${ }^{\mathrm{e}}$ siècle publiée par le grand vulgarisateur allemand Christoph Meiners en $1798^{29}$ dans le but de démontrer les

25. Voir infra les remarques de Wichelhausen sur la Révolution française.

26. Voir infra les remarques de Wichelhausen sur Alexandre; Lew Kopelew, « Deutsch-russische Wahlverwandtschaft », in Dagmar Herrmann, Alexander L. Ospovat, eds, Deutsche und Deutschland aus russischer Sicht. 19. Jahrhundert: Von der Jahrhundertwende bis zu den Reformen Alexanders II., Munich, 1998, p. 13-107, 104) considère les années 1800 à 1819 comme les meilleures dans les relations germano-russes du XIX ${ }^{\mathrm{e}}$ siècle.

27. Werner Schneiders, Die wahre Aufklärung. Zum Selbstverständnis der deutschen Aufklärung, Fribourg, 1974,p. 135, 171, 174 sq.

28. C'est une argumentation topique utilisée par exemple aussi par August Wilhelm Hupel, «Ueber den Nationalcharakter der Russen », Nordische Miscellaneen, t. 1, 1781, voir aussi H. Neuschäffer, « Unterschlagene Machtpolitik... », art. cit., p. 410-412.

29. Christoph Meiners, Vergleichung des älteren und neueren Rußlandes, in Rücksicht auf die Einwohner, Cultur..., Leipzig, 1798. 
changements de la Russie au siècle des Lumières. Cet ouvrage est la principale source «historiographique » utilisée, ce qui n'exclut pas que Wichelhausen ait lu Herberstein et Olearius dans la version originale ${ }^{30}$, ainsi que deux ouvrages statistiques sur l'Empire russe écrits par Benedikt Herrmann (de 1790) et l'économiste Heinrich Fr. Storch (1766-1835) de 1797-180331. Wichelhausen se démarque de la description très élogieuse de Moscou publiée par Richter en 179932. Les travaux les plus proches de sa propre entreprise sont la description de Pétersbourg par l'ethnographe Johann Gottlieb Georgi en $1790^{33}$ et une topographie médicale sur Hambourg, publiée par le médecin français Menuret en 179734. Les titres de ces trois derniers livres indiquent tous la difficulté de décrire une ville : les termes « esquisse » dans le titre de Richter et «essai »utilisé autant par Menuret que Georgi montrent l'insécurité épistémologique que ressentent ces auteurs. En même temps ces termes sont des marqueurs de la subjectivité de l'auteur ${ }^{35}$. Wichelhausen s'insère dans cette lignée en choisissant comme titre, Esquisse d'une peinture de Moscou $^{36}$. Lui aussi indique que sa description n'est pas complète et pourrait comporter à la fois des traits forcés ou trop faibles. Cette prudence dans la formulation du titre d'une part reflète la discussion sur les contenus nécessaires et superflus des topographies médicales ${ }^{37}$, d'autre part nous invite à considérer l'épistémologie générale de ce genre littéraire.

30. Il se réfère à eux parfois $(204,205)$.

31. Benedikt Franz Johann Herrmann, Statistische Schilderung von Rußland in Rücksicht auf Bevölkerung, Landesbeschaffenheit, Naturprodukte..., Sankt Petersburg, 1790; Heinrich Friedrich Storch, Historisch statistisches Gemälde des russischen Reiches am Ende des achtzehnten Jahrhunderts, 8 vols, Riga, 1797-1803 ; sur Storch, voir A. Grasshoff, « Heinrich Storch (17661835)...», art. cit., p. 111 sq.

32. Johann Gottfried Richter, Moskwa. Eine Skizze, Leipzig 1799; sur lui, voir Heike Joost, « Das Moskaubild Johann Gottfried Richters », in M. Keller, ed., Russen und Rußland aus deutscher Sicht. 19. Jahrhundert..., op. cit., p. 100-118 et E. Hexelschneider, Kulturelle Begegnungen zwischen Sachsen und Rußland 1790-1849, op. cit., p. 40, 44-50.

33. Johann Gottlieb Georgi, Versuch einer Beschreibung der russisch kayserlichen Residenzstadt Sankt Petersburg, Saint-Pétersbourg, 1790. Il avait en 1776-1781 publié une somme des grandes expéditions récentes, traduite de suite en anglais, russe et français, voir G. Robel, « Berichte über Rußlandreisen », art. cit., p. 236 sq.

34. Jean-Jacques Menuret (de Chambaud), Essai sur la ville d'Hambourg considérée dans ses rapports avec la santé ou lettres sur l'Histoire médico-topographique de cette ville, Hambourg, 1797. La traduction allemande (faite par Martin Gottfried Herrmann) paraît la même année sous le titre: Versuch über die Stadt Hamburg in Hinsicht auf die Gesundheit betrachtet oder Briefe über die medicinisch-topographische Geschichte der Stadt Hamburg.

35. Karl Riha, Die Beschreibung der « Großen Stadt». Zur Entstehung des Großstadtmotivs in der deutschen Literatur (ca. 1750-1850), Bad Homburg, Berlin, 1970, p. 45 sq., citant une lettre de Lichtenberg de 1775 et le titre d'une publication de Archenholz de 1787.

36. « Gemälde » est le terme allemand qui se rapproche du terme français « tableau » employé par Sébastien Mercier et d'autres auteurs et qui se réfère à un mode de perception de la ville entière ; voir Susanne Hauser, Der Blick auf die Stadt. Semiotische Untersuchungen zur literarischen Wahrnehmung bis 1910, Berlin, 1990, p. 104.

37. J. Brügelmann, Der Blick des Arztes..., op. cit., p. 29 sq. 


\section{La construction d'un objet : comment décrire une ville autour de 1800 ?}

Pour comprendre la construction de l'objet «ville» par Wichelhausen, une première approche consiste à considérer la table des matières comme un système de classification. Elle se présente comme suit :

\begin{tabular}{|c|c|c|c|}
\hline Numéro et titre du chapitre & $\begin{array}{c}\text { début } \\
\text { à la page }\end{array}$ & nb de pages & $\%$ de l'ouvr. \\
\hline I. Situation géographique de Moscou & 3 & 4 & 1,1 \\
\hline II. Regard sur l’histoire de Moscou & 7 & 15 & 4,1 \\
\hline $\begin{array}{l}\text { III. Description rapide des quartiers de M. ; } \\
\text { remarquables curiosités }\end{array}$ & 22 & 20 & 5,4 \\
\hline IV. Taille de la ville, habitat, voirie & 42 & 4 & 6,5 \\
\hline V. Les eaux de Moscou & 66 & 22 & 5,9 \\
\hline VI. Climat de Moscou & 88 & 47 & 12,7 \\
\hline $\begin{array}{l}\text { VII. Fertilité des sols, agriculture, } \\
\text { plantes autochtones et cultivées }\end{array}$ & 135 & 80 & 21,6 \\
\hline VIII. Élevage & 215 & 8 & 2,2 \\
\hline IX. Taille de la population & 223 & 12 & 3,2 \\
\hline X. Diversité des états & 235 & 48 & 12,9 \\
\hline $\begin{array}{l}\text { XI. État physique des corps, } \\
\text { dispositions intellectuelles et mentales }\end{array}$ & 283 & 27 & 7,3 \\
\hline $\begin{array}{l}\text { XII. Remarques générales sur la manière } \\
\text { de vivre, les mœurs et coutumes } \\
\text { des habitants de Moscou }\end{array}$ & 310 & 6 & 1,6 \\
\hline $\begin{array}{l}\text { XIII. La maison et la maisonnée } \\
\text { des habitants de Moscou }\end{array}$ & 316 & 25 & 6,8 \\
\hline XIV. Vêtements, toilette des dames & 341 & 15 & 4,1 \\
\hline $\begin{array}{l}\text { XV. Exercices physiques, } \\
\text { moyens de transport, voyages }\end{array}$ & 356 & 18 & 4,6 \\
\hline & & $371^{38}$ & 100,0 \\
\hline
\end{tabular}

Le texte traite en grande partie des questions qui sont communes aux topographies médicales. D'une part ces dernières suivent le questionnaire d'Hippocrate qui incitait à considérer les données environnementales exerçant une influence sur l'état de santé de la population : situation géographique du lieu (chap. I, III et IV), climat (VI), qualité des eaux (V) et des vivres (VII et VIII) et approvisionnement des deux ${ }^{39}$. Wichelhausen remplit ce cadre et inverse seulement l'ordre normal entre la description des eaux et du climat. D'autre part ces topographies traitent directement de l'état de santé des habitants, des maladies répandues ainsi que des professions et institutions qui sont chargées de les traiter. Ce deuxième centre

38. Le livre comporte 371 pages de texte sans les pages de titres et l'introduction, numérotées à part en chiffres romains.

39. J. Brügelmann, Der Blick des Arztes..., op. cit., p. 27. 
d'attention correspond à l'intérêt qu'a l'État à améliorer la santé publique par une meilleure administration de ce champ. Celui-ci se constitue autant par la systématisation des savoirs que par l'administration et la législation naissantes au XVIII ${ }^{\mathrm{e}}$ siècle ("medicinische Policey »)40. Les visées populationnistes des États, intéressés à développer la base physique de leur pouvoir, ressortent dans les chapitres IX et XI. Cet intérêt pour une population diverse, car composée de différents états (soslovija) et «nations » - Russes et non-Russes de l'Empire et étrangers -, mène aux descriptions des chapitres X à XII et permet d'intégrer le très traditionnel discours sur le «caractère national» des Russes, que Wichelhausen différencie selon des critères sociaux et ethniques. En même temps, il le recentre sur les aspects médicaux des « us et coutumes ». Le chapitre XIII sur la maison insiste sur les effets des constructions sur la santé. Les vêtements et la toilette des dames sont aussi traités selon cette optique, les effets dévastateurs du maquillage sur la santé et l'aspect « naturel » des femmes constituant un des sujets préférés des médecins des Lumières $^{41}$. La description des exercices physiques concerne, bien entendu, le remodelage du corps du « citoyen » qui devrait par ces pratiques se distinguer du noble typé de l'Ancien Régime, abusant des calèches et ne mettant jamais un pied devant l'autre - parfois même à l'intérieur de son appartement.

La table des matières montre que le texte de Wichelhausen correspond bien, en général, au genre de la topographie médicale et aux préoccupations typiques des médecins des Lumières. Sa relative pauvreté en informations sur les professions médicales le fait appartenir plutôt aux textes du dernier quart du XVIII ${ }^{\mathrm{e}}$ siècle qu'à ceux du premier tiers du XIX ${ }^{\mathrm{e}}$, dont le contenu devient plus technique et administratif après la parution de l'ouvrage de Frank ${ }^{42}$.

En même temps Wichelhausen participe à la longue tradition des descriptions ethnographiques (avant la lettre) de la Russie publiées par les voyageurs, personnes ayant résidé dans le pays ou dans des territoires annexés (pays baltes) pendant quelque temps. L'intérêt primordial de Wichelhausen pour les phénomènes environnementaux qui conditionnent la santé lui permet, sans forcer ce trait, de mieux organiser l'énorme masse de savoirs et d'éviter l'anecdotique qui caractérise souvent les rapports des voyageurs. On pourrait supposer que cette tradition de la description ethnographique l'a aussi inspiré aux chapitres II et III. Dans sa vision, l'histoire de la fondation de Moscou, des incendies et de la perte de sa fonction de capitale fait encore partie des savoirs nécessaire à la compréhension du présent. Wichelhausen donne aussi une description des quartiers de la ville qui comporte des informations sur l'aspect extérieur - colline du Kremlin opposée aux terrains plats et bas des autres quartiers - , les activités des habitants et même une liste des bâtiments les plus remarquables parmi lesquels se trouvent autant les monuments

40. M. Dinges, « "Medicinische Policey"... », art. cit.

41. Cf. Sabine Sander, «Der Arzt und die Kosmetik : Zerrbilder im Spiegel der deutschen Medizinhistoriographie », Medizinisches Journal, 37, 3/4, 2002, p. 265-300.

42. Johann Peter Frank, System einer vollständigen medizinischen Policey, 7 vols, Mannheim, puis Tübingen, puis Vienne, 1784-1819. 
historiques (palais, églises) que les institutions modernes d'enseignement (collèges, université), de santé (le fameux orphelinat, la pharmacie de l'opéra), de savoir (une bibliothèque, des archives et une imprimerie) et de production (les manufactures dans les faubourgs). Le patrimoine monumental de la ville se définit donc selon une pluralité de plans épistémologiques, l'histoire, la variété des confessions, le degré d'achèvement dans le processus pluriséculaire de formation des esprits, l'institutionnalisation de la santé publique et de la production.

Il est remarquable que Wichelhausen préfère asseoir sa description de la ville sur la traditionnelle répartition en quatre quartiers et 30 faubourgs, définis par le développement historique de la ville et leur localisation concentrique autour du Kremlin, à la division administrative en 20 secteurs principaux et 88 faubourgs de 1712 (23). La vieille classification serait mieux connue. Ce choix est certainement dû aussi aux exigences narratives du texte parce qu'il serait fastidieux d'énumérer une multitude de secteurs. Mais Wichelhausen montre éventuellement par là aussi une préférence pour un principe de géographie historique plutôt que pour le rationalisme du classement administratif, pourtant déjà introduit depuis trois générations. Ces décisions reflètent bien l'intention littéraire de l'auteur qu'on peut mettre en relation avec l'utilisation du mot « peinture » dans le titre du livre : les exigences du genre littéraire obligent Wichelhausen à se rapprocher - au niveau de la présentation de son objet - plutôt de l'art que de la statistique politique ou médicale. Par ailleurs il introduit des différences entre Moscou et Saint-Pétersbourg, Moscou et les petites villes russes ou le plat pays. L'objet « ville » est donc aussi constitué par une gradation à l'intérieur de la catégorie « ville ».

Wichelhausen définit la ville selon une multiplicité de plans épistémologiques ${ }^{43}$. Les uns ressortissent des sciences (géographie, météorologie, orographie, botanique), d'autres des sciences humaines naissantes (ethnographie, démographie) et de l'histoire humaine, d'autres des savoirs pratiques (agriculture, élevage). Les listes des bâtiments montrent l'inscription du pouvoir dans l'espace urbain autant par ses manifestations anciennes (Kremlin, églises et couvents orthodoxes) qu'actuelles (institutions modernes). Les seules références à l'économique concernent les bâtiments du marché et des manufactures et ultérieurement la production de vivres. L'économie n'est pas encore un élément de classification de plein droit.

Après ces remarques générales sur les systèmes de classification utilisés par Wichelhausen, voyons plus en détail son argumentaire sur la ville de Moscou.

\section{Premier argument : Moscou est une ville des Lumières comme toutes les autres}

Son premier argument implicite peut être formulé ainsi : «Moscou est une ville des Lumières comme toutes les autres ».

43. Cf. Bernard Lepetit, Les villes dans la France moderne (1740-1849), Paris, 1988, p. 123 et passim. 
La structure de la voirie serait comme dans d'autres grandes villes : les rues principales sont larges, et ne sont tortueuses que dans les vieux quartiers (58). La ville porte à travers ces vieilles rues le poids de son histoire (44), mais elle s'est ouverte à la modernisation de ses infrastructures. Moscou, en tant que grande ville, aurait les mêmes problèmes que d'autres villes : il y a peu de trottoirs, ce qui est dangereux pour les piétons quand des calèches ou traîneaux passent trop vite et causent ainsi des «accidents d'enfants, d'ivrognes et de sourds-muets » (60). Wichelhausen partage ce souci des accidents de rue avec Mercier ou Delamare ${ }^{44}$.

Sur d'autres plans Moscou serait une ville « bien policée » ${ }^{45}$ : la police veillerait parfaitement à la sécurité des rues grâce à la présence « d'hommes armés de matraques installés dans des huttes (les Butterschniki) » (63) qui mèneraient une lutte efficace contre les criminels. Le marché municipal serait bien organisé : son architecture est, selon Wichelhausen, bien fonctionnelle (32). La police lutte même de manière préventive contre le feu. En hiver le marché n'est ouvert que pendant les quelques heures où brille la lumière du soleil, parce qu'il est interdit d'allumer des bougies dans les boutiques. C'est là un « règlement très sage pour éviter des incendies » (33).

La police se chargerait aussi de l'ensemble des problèmes médicaux : ainsi « les cadavres des animaux sont enterrés, ce qui est un louable règlement de police » (219). Les Lumières ayant horreur de l'enterrement des êtres vivants, la remarque suivante se rapporte à cette phobie et comporte un compliment pour les institutions moscovites ${ }^{46}$ : «Les enterrements n'ont lieu qu'après production d'un certificat du chef de la police du quartier» (230). Après avoir découvert les effets des ruissellements souterrains des eaux, les Lumières en firent une autre de leurs grandes préoccupations en proposant, en bonne logique, d'éloigner les cimetières des centres villes. C'était une (petite) révolution culturelle que de séparer ainsi les morts des vivants. Pour Moscou, Wichelhausen constate que « les cimetières sont suffisamment loin des places habitées et que les habitants n'ont rien à craindre de leurs exhalaisons néfastes » (41). Dans cette ville, l'infrastructure souhaitable est déjà réalisée.

Pour ce qui est de la société moscovite, Wichelhausen y trouve des traits fort semblables aux sociétés du Centre- ou de l'Ouest européen : «Les vêtements des Russes du commun - hommes et femmes - sont plus adaptés à leur destination naturelle (!) et contribuent davantage à leur santé que les vêtements des gens nobles » (352). Connaissant la critique adressée à la noblesse par les Lumières, nous ne sommes pas étonnés de lire que les états supérieurs sont dégénérés (309) ${ }^{47}$.

44. S. Hauser, Der Blick auf die Stadt, op. cit., p. 113 sq. concernant Paris pendant les années 1710 .

45. Marc Raeff, The well-ordered police state : Social and institutional change through law in the Germanies and Russia, 1600-1800, New Haven, 1983.

46. Michel Vovelle, Mourir autrefois : attitudes collectives devant la mort aux XVII et XVIII ${ }^{e}$ siècles, Paris, 1983.

47. Roy Porter, « Modernité et médecine : le dilemme de la fin des Lumières », in Vincent Barras, Micheline Louis-Courvoisier, eds, La médecine des Lumières : tout autour de Tissot, Chêne-Bourg (CH), 2001, p. 5-24. 
Ces quelques exemples suffisent amplement pour illustrer le premier argument de Wichelhausen (à savoir que Moscou est une ville des Lumières comme toutes les autres). Ils montrent, par ailleurs, combien la grille de lecture qu'utilise Wichelhausen pour décrire Moscou est commune à tous les représentants des Lumières : nous retrouvons les préoccupations habituelles de son temps - voiries, accidents de circulation, éloignement des cimetières, lutte contre le feu, en somme la «ville bien policée » ainsi que la propagation des vêtements légers et la polémique contre la dégénérescence des états supérieurs... Bien sûr on pourrait y ajouter l'intérêt porté aux constructions, à la circulation de l'air, particulièrement dans les vieux quartiers et dans les maisons, et bien d'autres soucis... Par le biais de cette grille de lecture, Moscou devient un lieu des Lumières comme tous les autres et le lecteur contemporain des Lumières est, à Moscou, quasiment chez lui - comme à Berlin, Vienne ou Paris. Pour forcer un peu le trait, Moscou n'est pas seulement comparable à Vienne ou à Berlin, mais quasiment identique à ces deux villes.

\section{Deuxième argument : Moscou peut être placée sur l'échelle différentielle des villes russes et européennes}

Mais bien entendu Wichelhausen introduit des différenciations avec son deuxième argument : Moscou peut être placée sur l'échelle différentielle des villes russes et européennes.

La première comparaison qui s'impose inclut Saint-Pétersbourg, que Wichelhausen considère comme la plus belle ville d'Europe, dont les bâtiments ne seraient « pas surpassés par ceux du vieux Rome » (44). En matière d'architecture urbaine, Moscou, par contre, serait «définitivement la ville la plus étrange et la plus aventureuse » (44). Les bâtiments de Moscou offriraient un mélange de styles artistiques allant « du style gothique, [...] reliques des temps sombres [...] aux maisons et palais de style français plaisant », situés à côté de bâtiments « datant du temps des Mongols et d'autres peuples asiatiques » (44). Cette configuration ne forme pas un « ensemble harmonieux». La comparaison se fonde donc sur un argument esthétique.

D'autres catégories sont utilisées pour comparer les constructions : la densité et la hauteur moyenne des bâtiments seraient moindres à Moscou. Si on construisait dans cette ville comme à Londres ou à Paris, « Moscou dépasserait toutes les villes d'Europe en nombre d'appartements » (45). Moscou est donc impressionnante rien que par sa taille. Néanmoins l'avantage est qu'on y construit différemment parce que l'air circule mieux entre le grand nombre des petites maisons typiques de Moscou. «Les Russes tiennent les maisons de bois pour plus favorables à leur santé que les maisons en pierre », ce à quoi Wichelhausen souscrit «sous certaines conditions » (47) qu'il n'explicite pas. Mais il fait l'éloge de la simplicité du mode de construction de ces maisons à la manière des « anciens spartiates », exprimant par là un jugement de valeur fondé sur sa culture néo-classique et reprenant à son compte la préférence des Lumières pour la simplicité. 
Beaucoup de nouveaux palais en pierre «peuvent rivaliser avec ceux de Florence et de Rome » en ce qui concerne le goût architectural (51). Mais dans ces palais on « remarque qu'on n'est pas en Angleterre ni en Hollande ni dans un autre pays, où la solidité et la concordance entre l'extérieur et l'intérieur sont appréciées. Tout n'est fait que pour l'apparence extérieure, légère et point achevée dans toutes ses parties ». Dans sa géographie imaginaire Wichelhausen utilise l'Italie - considérée comme le pays par excellence de l'architecture de palais - comme le lieu de référence du bon goût. Pour la solidité architecturale, par contre, il se réfère plus volontiers aux pays du capitalisme avancé du Nord-Ouest européen. Moscou participe de manière différenciée aux avantages particuliers de ces deux Europes : l'imitation du goût paraît plus facile que l'appropriation de la partie technique. Ainsi l'aspect de ces palais moscovites reflète leurs propriétaires nobles - parfois nommément indiqués -, qui sont davantage tributaires de l'apparence aristocratique que de la substance bourgeoise du citoyen. Si la description de ces hôtels particuliers montre que quelques rares palais de l'ancienne capitale de la Russie peuvent exceptionnellement atteindre des sommets européens sur certains plans, en revanche, des pas restent à faire sur d'autres plans, plus significatifs parce qu'ils concernent les compétences techniques, valorisées par la bourgeoisie en tant que moyens indispensables pour maîtriser le futur.

Wichelhausen retrouve en partie, à propos des infrastructures publiques, la situation ambiguë qu'il a observée pour la construction privée. Il remarque que les « allées et boulevards sont semblables à ceux de Paris » (35). Mais l'approvisionnement des eaux reste plus problématique. D'une part «grâce à la police, l'eau de la Moskwa est moins polluée que dans le Berlin illuminé » (68). On voit dans cette pointe antiprussienne combien le mouvement des Lumières appelle, chez Wichelhausen, une action, sous l'égide de la police (médicale), visant à l'amélioration pratique des conditions de vie. Cette institution - à laquelle les médecins sont si sensibilisés à l'époque - aurait mieux réussi à Moscou qu'à Berlin ! Il n'empêche que l'approvisionnement naturel de l'ancienne capitale russe en eau est « un des plus médiocres de toutes les villes » (69). Des puits et des tuyaux de plomb ont amélioré la situation dans certains quartiers, mais les plus pauvres surtout sont très mal servis (76). Wichelhausen fait l'éloge de l'aqueduc commencé par Catherine II et conseille d'installer des puits filtrants comme à Paris ou à Amsterdam, qui pourraient aussi très bien servir à Venise. Pour chaque élément de ces infrastructures aquatiques, Wichelhausen établit des comparaisons entre Moscou et une ou plusieurs villes européennes.

Parfois Moscou se trouve en haut de l'échelle, parfois en bas, parfois au milieu, l'essentiel étant qu'on peut situer ainsi tout objet ou pratique sur une échelle commune qui intègre parfaitement cette ville à l'univers infiniment gradué des Lumières. Pour ne donner que quelques exemples supplémentaires de ce procédé, considérons le rendement en lait des vaches russes. Selon Wichelhausen, il serait très bas, parce que le fourrage serait moins bon qu'en Allemagne (218). Il vante, par contre, les exploits de l'horticulture moscovite : la ville de Moscou compterait dans son périmètre «le plus grand nombre de serres en Europe » et «les mieux 
équipées » (211). À Moscou il y aurait des centaines de serres, en Allemagne, en France ou en Italie seulement des exemples isolés (212).

À partir de là on n'est plus loin de l'éloge des avantages économiques dont profitent les étrangers à Moscou : les artistes et artisans assidus y vivraient « dans une plus grande aisance et feraient plus facilement fortune qu'en Allemagne» (253). Par conséquent leurs « filles portent des vêtements plus chers et plus élégants qu'en Allemagne et que dans beaucoup d'autres pays d'Europe » (344) ${ }^{48}$.

Dans la même logique comparative, Wichelhausen nous décrit aussi les objets culturels pour lesquels les Russes seraient à la pointe, si bien que les autres Européens devraient les imiter. Il s'agit surtout de l'art de chauffer observé à Moscou (328 sq.) : Wichelhausen décrit en détail l'architecture des poêles de brique. Il propose de les introduire dans les hôpitaux d'Europe $(331,336)$. La coutume de chauffer ces poêles avec du bois et de les fermer lorsque celui-ci s'est transformé en charbon permettrait d'économiser du bois et de produire une température régulière pendant 24 heures (336). Sans masquer les dangers techniques de certaines de ces procédures, Wichelhausen vante aussi l'habitude de chauffer plusieurs chambres en même temps (331 sq.), car la température régulière dans un appartement entier maintiendrait en bonne santé les occupants de la maison en leur évitant des changements trop rapides entre le froid et le chaud - dont les médecins ont horreur depuis Hippocrate $^{49}$. Il en fournit personnellement la preuve en citant des cas de malades allemands guéris à Moscou de leurs catarrhes grâce à ces manières de chauffer et, pour renforcer cet argumentaire, en y ajoutant sa propre expérience de sept années de vie en Russie où les bâtiments étaient toujours bien chauffés, par opposition à Venise où il avait eu très froid pendant sa visite en janvier $1794(328,329)$.

Le deuxième succès civilisateur des Russes, digne d'être imité, serait constitué par les «kibitki». Sans entrer dans le détail, précisons qu'il s'agit de carrosses fermés et très confortables permettant de voyager allongé sur un matelas. On pouvait même y passer la nuit, ce qui évitait les lits d'hôtels miteux que chaque voyageur connaissait partout en Europe ainsi que le risque d'y contracter des maladies (368-372).

La possibilité de situer sur une échelle graduée tous les objets et phénomènes observables s'applique aussi à l'état physique des Russes : les femmes russes de Saint-Pétersbourg et de Moscou seraient plus fertiles que les étrangères vivant sous le même climat. Wichelhausen explique ce phénomène par la dégénérescence des immigrées, affaiblies, tout au long des générations antérieures (232), par les influences de la culture occidentale raffinée qui continueraient à se faire sentir pendant leur séjour en Russie. Pour ce qui est des femmes russes, le statisticien allemand Storch avait prétendu qu'elles étaient pubères plus tôt que les étrangères, expliquant cette précocité par leur usage des bains de vapeur (289). Wichelhausen

48. Sur les appartements plus somptueux des classes moyennes, voir E. Wichelhausen, Züge zu einem Gemählde von Moskwa..., p. 329.

49. Cet argument est à rapprocher de l'évocation des propriétés isolantes des calèches russes par rapport au froid. 
conteste cette opinion en utilisant la comparaison entre les Russes et les étrangères vivant à Moscou, qui n'avaient pas eu recours à ces mêmes bains mais étaient néanmoins pubères aussi jeunes que leurs congénères russes. La raison serait donc à chercher ailleurs, notamment dans le climat local qui pousserait les plantes et les animaux à parcourir plus rapidement les diverses phases de la croissance et qui aurait une action analogue sur les êtres humains ${ }^{50}$. L'Amérique du Nord est utilisée comme preuve (290). Ceci n'empêche pas Wichelhausen de prétendre, cinq pages plus bas, que les étrangers - donc hommes et femmes - continueraient de vivre à Moscou comme dans leur pays d'origine, si bien que le climat ne pourrait pas les changer fondamentalement (295). L'argumentation paraît donc comporter une contradiction entre la thèse du changement par le climat et celle d'une limite « génétique » à ce même changement.

Dans sa tentative pour saisir l'état physique des femmes russes, Wichelhausen répète différentes idées clés des Lumières sur les peuples jeunes et les peuples vieux - donc dégénérés - et sur les effets du climat. Il considère que l'influence de ce dernier est plus grande que celle de la coutume locale des bains. Il choisit pour méthode de comparer deux échantillons (quasi statistiques) de femmes russes et étrangères. L'argument de l'importance du climat est souligné et vérifié par la référence à l'Amérique. Le climat créerait donc seulement une spécificité pour chaque zone climatique ${ }^{51}$. Ainsi les Russes se retrouvent une fois de plus situés dans le monde imaginaire des Lumières, cette fois dans cette «autre Europe » qu'est l'Amérique. Tout de même, ces rapprochements ont des limites. Si le climat peut causer une puberté précoce pour toutes les femmes vivant à Moscou, il ne peut changer génétiquement les étrangères, parce qu'elles vivent - selon Wichelhausen - moins sous son influence. Son approche foncièrement comparative trouve donc une limite significative dans des propriétés inhérentes aux individus ou aux groupes, appelées dans ce contexte « génétiques ». «Génétique » pourrait signifier «toute maladie qui concerne d'abord les fonctions du sexe» englobant les «désordres des sécrétions, les anomalies des poussées sexuelles et les gravidités maladives » selon le système développé par John Mason Good (1764-1827) mais postérieur de quelques années au texte de Wichelhausen ${ }^{52}$, qui, lui, fait bien référence au moment de la puberté. Ce que la médecine définit sous le terme de première menstruation, ce sont bien les changements des « sécrétions et des poussées sexuelles ». Mais le terme « génétique » paraît indiquer au-delà d'un concept médical des résonances avec les idées de Herder (1744-1803) supposant une substance particulière

50. Un phénomène parallèle est analysé chez les hommes à propos de la formation de la barbe (286).

51. Pour le contexte de ce débat, cf. Michael A. Osborne, « The geographical imperative in nineteenth-century French medicine », in A. Rupke, ed., Medical geography in historical perspective, op. cit., p. 31-50, 32-34.

52. Friedrich Ludwig Meissner, ed., Encyclopädie der medicinischen Wissenschaften nach dem Dictionnaire de médecine, vol. 5, Leipzig, 1831 ; voir A. Hirsch, Biographisches Lexikon der hervorragenden Ärzte aller Zeiten und Völke, op. cit., vol. 2, p. 797 sur Good et les dates de publication de son œuvre. 
propre aux peuples, et qui, par conséquent, pourrait aussi se retrouver dans le corps de chaque membre de tel ou tel peuple ${ }^{53}$. Wichelhausen utilise le terme «génétique » pour caractériser la provenance (ethnique) de la population entière (sans les nobles considérés comme le produit de mélanges de peuples immigrés) (283) et pour expliquer le petit nombre de bossus dans la population des moujiks - à la différence de celle de la ville allemande de Hambourg (291) — en se référant à la « race » comme élément explicatif (292). Il voit dans la tendance des enfants russes à crier la manifestation d'une grande «force vitale génétique »(292). Le génétique est donc une qualité innée d'un peuple qui le différencie des autre peuples, mais qui peut être modifiée partiellement par le climat et le mode de vie sans toutefois disparaitre complètement pendant une génération.

La difficulté à mener jusqu'au bout la comparaison entre Moscou et les autres villes des Lumières mène au troisième argument de Wichelhausen : « Moscou est foncièrement différente ».

\section{Troisième argument : Moscou est foncièrement différente}

Cela pourrait être illustré par les remarques de Wichelhausen - citées supra — sur le patrimoine bâti qui rappellerait parfois les héritages mongols. Le terme courant marquant cette différence est le «goût oriental ». Chez Wichelhausen, cela ne dénote par ailleurs rien de péjoratif. Il décrit même les avantages d'un habitat espacé construit selon ce goût qui laisserait circuler l'air plus librement (46).

Mais c'est bien entendu à propos du servage qu'il lui est possible d'affirmer une différence totale entre la Russie et l'Europe ${ }^{54}$. Ce propos est incontournable dans la description de la ville, qui serait foncièrement imprégnée dans toutes ses fibres et ses conditions d'existence par ce système. Ceci se ressent par exemple en matière d'approvisionnement des vivres, quand on considère les faibles rendements dus au « système du servage qui pèse sur toute l'agriculture de la Russie » et qui la différencie de « l'Europe entière » (143). L'ethnographie de la ville ne peut se distinguer sur ce sujet d'une description de la Russie qu'en précisant les traits urbains du

53. Sur Herder et sa représentation des Russes, voir D. Groh, Rußland und das Selbstverständnis Europas..., op. cit., p. 70-80 ; Mechthild Keller, «'Politische Seeträume' : Herder und Rußland », in M. Keller, ed., Russen und Rußland aus deutscher Sicht. 18. Jahrhundert..., op. cit., p. 357-395; sur sa notion de peuple et les différences de celle-ci par rapport au concept de Schlözer, voir Justin Stagl, A history of curiosity : The theory of travel 1550-1800, Chur, 1995, p. 255 sq., 267. L'idée (inverse) que le climat créerait des corps plus résistants se trouve par exemple chez Bielfeld en 1773, cf. H. Klueting, « Rußland in den Werken deutscher Statistiker des 18. Jahrhunderts », art. cit., p. 255.

54. Sujet de débat en Russie, voir Erich Donnert, « Aufklärung und antileibeigenschaftliches Denken in Rußland in der zweiten Hälfte des 18. Jahrhunderts », Zeitschrift für Geschichtswissenschaft, 20, 1972, p. 974-986, et à l'étranger, H. Neuschäffer, «Unterschlagene Machtpolitik... », art. cit., p. 430 ; pour une comparaison récente, voir Christoph Schmidt, Leibeigenschaft im Ostseeraum. Versuch einer Typologie, Cologne-Weimar, 1997 ; appréciation de sa valeur économique chez Roger Bartlett, «Die Rationalität der Leibeigenschaft in Rußland in der Regierungszeit Katharina II. », in Claus Scharf, ed., Katharina II., Rußland und Europa, Mayence, 2001, p. 403-419, 419. 
phénomène connu par ailleurs. On pourrait paraphraser les descriptions différenciées des conditions de vie des « moujiks » que Wichelhausen excelle à livrer dans son texte. Elles ont bien sûr tout leur intérêt dans une topographie médicale. Mais ces individus qui préfèrent se laisser mourir plutôt que de prendre des médicaments pour ne pas attirer la honte sur leur seigneur (264) ne font qu'illustrer les effets dégradants du servage. Le terme "asiatique » est l'expression évocatrice utilisée par Wichelhausen pour caractériser l'habitude des nobles de se faire accompagner par une multitude de serfs (53/54). Ce comportement lié à la vanité inciterait les aristocrates à exagérer le nombre des membres de leur escorte pour lesquels ils ne peuvent ni ne veulent payer des logements décents. Et on apprendrait des détails sur la «hutte noire» (Schwarzstube), le taudis de bois noirci de fumée, humide, surpeuplé d'occupants misérables où logent ces malheureux (53). Tous ces détails ne font qu'illustrer l'argument de la différence fondamentale de la Russie, en même temps qu'ils remettent le servage en question.

Néanmoins, Wichelhausen introduit sur deux plans des nuances dans cette vision. D'une part il compare le servage en Russie avec celui des « esclaves de Rome et de Sparte » et celui des esclaves noirs «dans les deux Indes » qui, tous, auraient souffert davantage. Ce serait aussi le cas des serfs lettons (253) ${ }^{55}$. Dans les deux comparaisons, la Russie se démarque par un traitement plus humain qui peut aussi être interprété comme une avancée historique. D'autre part Wichelhausen dessine les traits de la personnalité des «moujiks », qui seraient modelés par une vie monotone (307). Ceci aurait aussi des répercussions sur le train de vie de tous les Moscovites. La «diversité moindre des états» y introduirait une certaine langueur. La ville souffrirait donc du servage. Les serfs de la ville seraient par ailleurs souvent habiles et gentils. Wichelhausen les présente comme « ayant une grande ressemblance avec le tempérament des Français » $(307)^{56}$ et comprenant plus rapidement que le paysan allemand une prescription médicale (302). Malgré les effets destructeurs du servage, les hommes ne sont donc pas (encore) perdus. Il se peut que Wichelhausen ne veuille pas surestimer le système en soi et souhaite considérer d'abord les individus. En somme les moujiks seraient donc parfaitement éducables et disposés à recevoir une formation inspirée des Lumières.

Certes, Wichelhausen dénonce vivement - et volontairement (voir XIII et supra) - les abus du servage et constate par ce moyen une différence fondamentale entre la Russie et l'Europe. Mais il nuance fortement ses propos en introduisant des comparaisons favorables à la Russie sans nier la différence. Il démontre que son potentiel humain devrait être libéré57. Il l'illustre avec l'exemple des moujiks « venus de l'intérieur de la Russie, assidus, économes et abstinents », qui opèrent

55. Sur le mythe, consciemment répandu par Catherine II, d'une situation encore plus déplorable des serfs livoniens, qui se trouve développé dans un livre de Eisen remanié par la censure russe qui y ajouta un chapitre supplémentaire, voir H. Neuschäffer, «Unterschlagene Machtpolitik... », art. cit., p. 416.

56. Cette comparaison permet à Wichelhausen de lancer une pointe contre les voyageursécrivains français.

57. Dans l'image positive de la population russe, il suit Georgi, une de ses sources (voir supra). 
de grandes réussites dans le jardinage à Moscou (207). Ne sont-ils pas de paradigmatiques « icônes » du changement, sortis des ténèbres de l'espace russe, mais incarnant de manière étonnante toutes les vertus bourgeoises par excellence ? En tout cas, Wichelhausen n'utilise pas sa description du servage pour étayer la thèse d'un retard structurel russe qui agirait comme un cercle vicieux dans lequel le servage crée le retard qui, à son tour, maintient le servage ${ }^{58}$. C'est plutôt le contraire : Wichelhausen est optimiste sur les chances de libération du servage. On ressent l'inspiration puisée chez Herder selon laquelle il existerait des potentialités chez les peuples slaves. Cette inspiration se double d'une vision positive des forces cachées dans les classes populaires que Wichelhausen partage avec d'autres représentants des Lumières. De là se comprend son quatrième argument: Moscou comme témoignage du changement de la Russie.

\section{Quatrième argument : Moscou comme témoignage des chances de changement de la Russie}

La «profondeur du sentiment religieux des Russes » est une idée reçue qui fait, encore de nos jour, bien vendre les livres sur la Russie dans «l'Occident». En même temps la relation des représentants des Lumières à la religion est connue ${ }^{59}$. La description de la vie religieuse dans un texte de l'époque est un bon indicateur d'un changement culturel en profondeur. Écoutons Wichelhausen dénoncer les pratiques religieuses des Russes, qu'il considère comme dangereuses pour des raisons tout à fait « illuminées » : selon lui tous les Russes allumeraient des bougies devant les images des saints qui se trouvent dans les antichambres. Par manque de moyens, les moins aisés le feraient seulement les jours fériés. Néanmoins cet usage aurait souvent abouti à des incendies. Maintenant, par contre, «cela n'arrive que rarement »(328).

Il y a donc un changement qui peut autant résulter d'une moindre ferveur dans la pratique religieuse que d'un accroissement de la prudence des pratiquants. Même dans le deuxième cas, le religieux serait littéralement civilisé, donc endigué car entouré de conscience pratique moderne. Peu importe, l'essentiel est le changement vers un mieux selon les critères de l'auteur. On a déjà constaté ce même phénomène dans les remarques faites à propos des progrès dans l'administration de la police. C'est au style des publications triomphantes de la fin du XVIII ${ }^{e}$ siècle qui voulaient rendre compte des avancés réalisées pendant le siècle des Lumières, que nombre des récits de Wichelhausen empruntent leur structure. Les habitants et les autorités de Moscou sont donc, sur plusieurs plans, en marche vers un avenir meilleur (ou radieux).

58. Manfred Hildermeier, «Das Privileg der Rückständigkeit. Anmerkungen zum Wandel einer Interpretationsfigur der neueren russischen Geschichte », Historische Zeitschrift, 244, 1987, p. 557-603.

59. Le rapport des gouvernants à la religion et aux églises est très important dans le jugement des Lumières sur la Pologne et la Russie, voir I. Hanslik, Das Bild Rußlands und Polens im Frankreich des 18. Jahrhunderts, op. cit., p. 301. 
Pour le décrire Wichelhausen utilise le découpage en quatre périodes qui organise habituellement les descriptions de la Russie : avant Pierre le Grand ; le règne de Pierre le Grand ; la période après Pierre jusqu'au tsar/à la tsarine réformateur/ trice contemporain/e (actuellement Alexandre); la période actuelle pleine d'espoirs grâce aux réformes commencées ou espérées de ce tsar éclairé ${ }^{60}$. Il n'est pas étonnant que Wichelhausen propose tout de suite toutes les bonnes recettes du changement allant de l'exemple individuel à la législation impériale. Les bonnes lois jouent bien entendu un rôle crucial. Wichelhausen énumère les éléments structurels et personnels qui pourraient empêcher ou promouvoir le développement : un style de vie moins rustre et la dissémination des savoirs (321). Wichelhausen propose à ce titre des améliorations pour la publication de textes utiles (220). Il considère les forces de changement autochtones et étrangères au pays. Implicitement il livre une psychologie du changement possible en s'inspirant du lent apprentissage que les Moscovites, depuis le tsar Pierre, auraient fait en s'habituant à voir des vêtements étrangers (343). Il veut donc respecter le rythme originel du peuple concerné, tout à fait dans la tradition de Montesquieu (1689-1755) et de Herder, lorsqu'il s'interroge sur les expériences plus récentes de la Révolution française $(273 / 274)^{61}$. Il insiste plus d'une fois sur la nécessaire lenteur des changements, ce qui démontre une position prudente par ailleurs très répandue en $1803^{62}$. Il semble qu'au-delà des considérations politiques générales de son temps, Wichelhausen veut marquer, par son insistance sur la lenteur nécessaire du changement, une autre spécificité russe liée aux effets du servage.

\section{Conclusion}

On a passé en revue les multiples classifications différenciées qui structurent la topographie médicale de Wichelhausen. Les possibilités et les limites de ce genre littéraire ont été mises en valeur. On a vu la difficulté de construire «la ville» comme objet pertinent et la complexité des plans épistémologiques mobilisés dans cette construction. Cette construction d'objet ne met pas l'auteur à l'abri de la tentation de réintroduire des généralités sur « les Russes » tirées d'autres sources.

Il est devenu évident que l'auteur utilise ses savoirs de représentant des «meilleures » Lumières, de médecin « rationnel» - donc « scientifique » - et d'Allemand. Cette triple identité marque sensiblement la structure et le contenu de son livre. Le texte contient d'abord et surtout des informations sur les préférences culturelles de son auteur, preuve qu'il est difficile de ne pas prendre « ses propres mœurs comme mesure et échelle pour la description de la Russie », comme Wichel-

60. Des éléments de ce modèle semblent continuer à exister dans les appréciations des nouveaux présidents de la Russie par l'opinion publique actuelle.

61. Herder voulait éduquer peu à peu («allmählich heraufbilden ») les peuples, cf. M. Keller, «'Politische Seeträume' : Herder und Rußland », art. cit., p. 377.

62. Cf. W.Schneiders, Die wahre Aufklärung. Zum Selbstverständnis der deutschen Aufklärung, op. cit., p. 174. 
hausen le reprochait aux écrivains-voyageurs (IX, voir supra) dans son introduction. Le texte de Wichelhausen ne présente qu'un autre système de classification, celle des Lumières médicales dans leur variante allemande. On peut bien le caractériser comme une collection de préjugés spécifiques (ou - si l'on préfère - scientifiques).

On peut se demander si cette collection de préjugés permet une valorisation de la culture de l'autre ${ }^{63}$. Wichelhausen tente sur deux plans cette valorisation. D'une part, sa grille de lecture intègre la ville de Moscou à ses propres échelles de valeur, d'autre part, elle permet - et l'auteur s'y autorise - l'affirmation d'une différence fondamentale. Il est intéressant de noter que cette différence, qui se situe autour de 1803, repose sur l'histoire de la ville, la génétique des corps, le servage et la lenteur du changement que l'auteur préconise en partie.

Néanmoins Wichelhausen nous propose une image de Moscou et des Russes qui est quadruple : ils seraient premièrement comme les autres Européens, deuxièmement comparables aux Européens, troisièmement différents et, quatrièmement, ils auraient finalement un grand potentiel à développer selon leur propre rythme. Ils seraient alors divers et toujours à redécouvrir. Ce n'est pas un mauvais message même s'il est basé sur un solide fonds de préjugés datant de 1800.

\section{Institut für Geschichte der Medizin der Robert Bosch Stiftung \\ Straußweg 17 \\ D-70184 Stuttgart}

63. En dehors de l'analyse interne du texte proposée ici, la comparaison avec d'autres textes du même genre serait la condition nécessaire d'une appréciation de la valeur spécifique du système de classification utilisé par Wichelhausen pour comprendre et présenter une culture étrangère. Comme texte comparable on pourrait prendre d'une part une topographie médicale d'une autre ville russe (Saint-Pétersbourg) considérée comme plus moderne et/ou européenne par certains auteurs, dont Wichelhausen se démarque par sa vision relativement positive de Moscou. On pourrait d'autre part choisir une ville allemande décrite par un étranger (même genre littéraire, différence dans le lieu décrit et la nationalité de l'auteur), une topographie médicale de Pétersbourg ou une description de la ville par un autre spécialiste (statisticien par exemple) et d'autre part des descriptions de non-médecins de Moscou. Une première analyse de la topographie médicale du médecin français Menuret (voir note 34) sur Hambourg paraît indiquer que l'auteur constate un contraste nettement moins grand entre cette ville d'Allemagne et des villes françaises - décrites par lui antérieurement - que celui que décrit Wichelhausen entre l'Allemagne et la Russie. Pour la construction des différences, Menuret utilise d'autres indicateurs scientifiques (climatiques...) et culturels - tels que le caractère supposé (flegmatique - lié au climat), la nourriture et les formes de repas et les manières de se couvrir pendant le sommeil. Une autre question que l'on ne s'est pas posée dans cet article est celle de la diffusion et de la réception du texte de Wichelhausen, qui nécessiterait en premier lieu une recherche sur les comptes rendus. Et bien entendu, la question de l'expérience de la ville peut être traitée de manière très différente, voir Sadowsky et Harald Tersch, « Die Kategorisierung des Blicks. Städtische Identität in Wien-Berichten der frühneuzeitlichen Reiseliteratur », FrühneuzeitInfo, 10, 1999, p. 108-133. 